\title{
Concentração de metais pesados em espécies arbóreas utilizadas para revegetação de área contaminada ${ }^{1}$
}

\author{
Heavy metals concentration in tree species used for revegetation of contaminated area
}

\author{
Ana Carolina Callegario Pereira ${ }^{2 *}$, Ana Carolina Dornelas Rodrigues ${ }^{2}$, Fabiana Soares dos Santos ${ }^{3}$, Jair do \\ Nascimento Guedes ${ }^{4}$ e Nelson Moura Brasil do Amaral Sobrinho ${ }^{4}$
}

\begin{abstract}
RESUMO - O objetivo deste trabalho foi avaliar o potencial das espécies arbóreas Cordia africana Lam., Mimosa caesalpineafolia Benth., Acacia angustissima (Mill.) Ktze. e Anadenanthera colubrina (Vell.) Brenan na fitoestabilização de metais pesados presentes em um substrato contaminado. Foram selecionados, aleatoriamente, quatro exemplares de cada espécie para realização do estudo. Amostras de substrato na projeção da copa de cada exemplar arbóreo foram coletadas nas profundidades de 0-10; 10-30 e 30-50 cm, para determinação da concentração pseudototal e fracionamento químico de metais pesados. De cada indivíduo, foram coletados dados referentes à profundidade das raízes, DAP (diâmetro a altura do peito), diâmetro de copa e altura das plantas. Amostras de folha, caule, casca e raiz foram coletadas para determinação dos metais pesados zinco $(\mathrm{Zn})$, manganês $(\mathrm{Mn})$, cádmio $(\mathrm{Cd})$, chumbo $(\mathrm{Pb})$ e cobre $(\mathrm{Cu})$ nas diferentes partes da planta. A concentração de Cd no substrato foi acima do valor de investigação definido pelo CONAMA $\left(3,0 \mathrm{mg} \mathrm{kg}^{-1}\right)$, evidenciando contaminação com esse elemento. Os maiores teores dos metais pesados foram encontrados na fração residual (F5), indicando baixa disponibilidade destes elementos no substrato. Com relação ao Cd, elevadas concentrações também foram encontradas na fração trocável F2, considerada uma fração em que os contaminantes se apresentam com certa mobilidade e biodisponibilidade. As espécies vegetais estudadas apresentaram desenvolvimento dentro dos padrões normais, mostrando tolerância a áreas contaminadas com Cd, que tendeu a se acumular nas raízes das plantas, com exceção da Córdia - africana.
\end{abstract}

Palavras-chave: Arborização. Revegetação. Solos-descontaminação.

\begin{abstract}
This study aims to evaluate the potential of tree species Cordia africana Lam., Mimosa caesalpineafolia Benth., Acacia angustissima (Mill.) Ktze. and Anadenanthera colubrina (Vell.) Brenan in the phytostabilization of heavy metal from a substrate contaminated. Four specimens of each tree specie were randomly determined for evaluations. Substrate samples were collected in the projection of the canopy trees of each specimen at depths 0-10, 10-30 and 30-50 cm for determining pseudototal concentration and chemical fractionation of heavy metals. Were collected data on roots depth, diameter at breast height, canopy diameter and plant height for each specimen. Samples of root, stem, bark and leaf were collected for analysis of heavy metals zinc $(\mathrm{Zn})$, manganese $(\mathrm{Mn})$, cadmium $(\mathrm{Cd})$, lead $(\mathrm{Pb})$ and copper $(\mathrm{Cu})$. The $\mathrm{Cd}$ concentration in the substrate was above the investigation value defined by CONAMA $\left(3,0 \mathrm{mg} \mathrm{kg}^{-1}\right)$, showing contamination with this element. However, the highest concentrations of other studied heavy metals were found in the residual fraction (F5), indicating low availability of these elements in the substrate. High concentrations of $\mathrm{Cd}$ were also found in the exchangeable fraction $\mathrm{F}$ 2, which is considered a fraction in which contaminants are present with some mobility and bioavailability. The plants showed development within the normal patterns, showing tolerance to Cd-contaminated areas, which tended to accumulate in the roots, except in the Córdia - africana.
\end{abstract}

Key words: Afforestation. Revegetation. Soils-decontamination.

\footnotetext{
*Autor para correspondência

${ }^{1}$ Recebido para publicação em 04/02/2011; aprovado em 11/04/2012

Parte da Tese de Doutorado da primeira autora defendida no Programa de Pós-Graduação em Agronomia/Ciência do Solo/UFRRJ

${ }^{2}$ Departamento de Formação Profissional do Centro de Tecnologias e Engenharias/UniFOA-Centro/Universitário de Volta Redonda/Volta Redonda, Rio de Janeiro, Brasil, acallegario@yahoo.com.br, kroldornelas@yahoo.com.br,

${ }_{3}^{3}$ Departamento de Engenharia de Agronegócios/Universidade Federal Fluminense/Volta Redonda, Rio de Janeiro, Brasil, fabianasoares@id.uff.br

${ }^{4}$ Departamento de Solos/Universidade Federal Rural do Rio de Janeiro, Seropédica, Rio de Janeiro, Brasil, jgrural@yahoo.com.br, nelmoura@ufrrj.br
} 


\section{INTRODUÇÃO}

Pesquisas envolvendo a utilização de plantas em estratégias de fitorremediação têm aumentado nos últimos anos, sendo esta considerada uma alternativa emergente na recuperação de áreas contaminadas (KAVAMURA; ESPOSITO, 2010; PAQUIN; CAMPBELL; LI, 2004; XIAO etal., 2008;). Entre as estratégias de fitorremediação, a fitoestabilização (que consiste no uso de plantas visando estabilizar os poluentes no solo) pode ser muito útil também para prevenir perdas de nutrientes e metais pesados por erosão ou lixiviação (PILON-SMITS, 2005).

Entre os vegetais, as espécies arbóreas são frequentemente associadas à estratégia de fitoestabilização, sendo que a localização de metais nas raízes e a baixa translocação para a parte aérea têm sido relatadas como mecanismos de resistência de espécies arbóreas a metais pesados (PULFORD; WATSON, 2003). As árvores tendem a reduzir a lixiviação, controlar a erosão e adicionar matéria orgânica ao solo, o que pode levar à complexação dos metais pesados. Por outro lado, as árvores também podem acidificar o solo e produzir matéria orgânica solúvel podendo causar a lixiviação de metais. Diante disso, é importante a seleção de espécies arbóreas que causem baixa acidificação do solo e não transloquem elevadas quantidades de metais pesados para suas folhas (NEVEL et al., 2007).

O desenvolvimento de espécies arbóreas pode induzir várias mudanças nas características do solo, representando importante papel na ciclagem e fracionamento de metais pesados, devido à produção de liteira, exsudados radiculares e/ou absorção e imobilização na planta, principalmente no sistema radicular (BRUNNER et al., 2008).

A estabilidade genética da tolerância de espécies arbóreas a metais pesados é questionável, pois esta pode ser induzida ou perdida pelas plantas, sendo que a aclimatação das árvores no ambiente contaminado é de fundamental importância e pode ser um fator decisivo no seu desenvolvimento em solos contaminados (PULFORD; WATSON, 2003). Desta forma, estudar espécies que já tenham apresentado desenvolvimento satisfatório em condições características de cada região funciona como um acelerador nos resultados de pesquisas, visto que a etapa de avaliação de adaptabilidade já foi ultrapassada.

O objetivo do trabalho foi realizar a avaliação do potencial das espécies arbóreas Córdia - africana (Cordia africana Lam.), Sabiá (Mimosa caesalpineafolia Benth.), Acácia (Acacia angustissima (Mill.) Ktze.) e Angico branco (Anadenanthera colubrina (Vell.) Brenan) na fitoestabilização de metais pesados presentes em um substrato contaminado na Ilha da Madeira, município de Itaguaí - RJ.

\section{MATERIAL E MÉTODOS}

O experimento foi realizado em uma área de aproximadamente oito hectares localizada no município de Itaguaí - RJ (22\%47' de latitude sul e 4340' de longitude oeste), próxima a Baía de Sepetiba. Devido à sua proximidade ao Porto de Itaguaí, essa área serviu como suporte à deposição de substratos retirados no processo de dragagem da Baía de Sepetiba para aprofundamento do calado do Porto. Após deposição, esses substratos foram classificados como Classe II A (não inerte) devido aos teores de Cd estarem acima dos níveis permitidos pelo teste de solubilidade, segundo Associação Brasileira de Normas Técnicas (2004). A disposição do substrato e a revegetação da área foram realizadas em 1999, sendo que a deposição foi heterogênea e fez com que o aterro apresentasse profundidades diferentes, podendo atingir até $1 \mathrm{~m}$.

Sete anos após a revegetação, foi realizado o levantamento botânico das espécies existentes na área e foram selecionadas as que apresentavam melhor desenvolvimento, levando em consideração o número de exemplares e a altura das plantas. As espécies selecionadas para o estudo foram: Córdia - africana (Cordia africana Lam.) Família Boraginaceae, Sabiá (Mimosa caesalpineafolia Benth.) Família Fabaceae-Mimosoideae, Acácia (Acacia angustissima (Mill.) Ktze.) Família Fabaceae-Mimosoideae e Angico branco (Anadenanthera colubrina (Vell.) Brenan) Família Fabaceae-Mimosoideae. Foram determinados, aleatoriamente, quatro exemplares de cada espécie para a realização do estudo.

Amostras de substrato, na projeção da copa de cada exemplar arbóreo, foram coletadas na profundidade de 0-20 cm. Essas amostras foram secas ao ar, destorroadas e passadas em peneira de $2 \mathrm{~mm}$ de malha de nylon para determinação de $\mathrm{pH}$ em água $(1: 2,5)$, análise de atributos químicos para fins de fertilidade e análise granulométrica, segundo Empresa Brasileira de Pesquisa Agropecuária (1997).

Para análise de metais pesados $(\mathrm{Zn}, \mathrm{Mn}, \mathrm{Cd}, \mathrm{Pb}$ e $\mathrm{Cu}$ ), amostras de substrato nas profundidades de 0-10; 10-30 e 30-50 cm, representando a profundidade onde foi observada uma maior quantidade de raízes, foram coletadas, trituradas em almofariz de ágata e passadas em peneira de malha de nylon, com abertura de $210 \mu \mathrm{m}$. Os teores pseudototais foram obtidos por meio da digestão nitro-perclórica 2:1, segundo Scott (1978) e quantificados por espectrofotometria de absorção atômica.

$\mathrm{O}$ fracionamento químico desses metais foi realizado utilizando metodologia proposta por Santos et al.(2007)com pequenas adaptações, que consiste em extrações simples, utilizando-se as seguintes soluções extratoras: água, cloreto de magnésio, DTPA e ácido acético. A partir dos teores de $\mathrm{Zn}, \mathrm{Mn}, \mathrm{Cd}, \mathrm{Pb}$ e $\mathrm{Cu}$ obtidos nas extrações simples, foram estimadas, por meio das diferenças nas quantidades extraíveis, frações químicas que consistiram de: 
$\mathrm{F}_{1}=$ Extraído com água $=>$ Fração solúvel em água.

$\mathrm{F}_{2}=$ Extraído com $\mathrm{MgCl}_{2}$ - Extraído com água $\Rightarrow$ Fração trocável, que compreende os metais ligados eletrostaticamente às partículas do solo.

$\mathrm{F}_{3}=$ Extraído com DTPA - Extraído com $\mathrm{MgCl}_{2}=>$ Fração predominantemente associada a óxidos de $\mathrm{Fe}$ e $\mathrm{Mn}$ mais cristalinos e a compostos orgânicos mais estáveis através da formação de complexos de esfera interna.

$\mathrm{F}_{4}=$ Extraído com ácido acético - Extraído com $\mathrm{MgCl}_{2} \Rightarrow$ Fração associada predominantemente a carbonatos e a óxidos de Mn amorfos pela formação de complexos de transição.

$\mathrm{F}_{5}=$ Pseudototal $-\sum(\mathrm{F} 1+\mathrm{F} 2+\mathrm{F} 3+\mathrm{F} 4) \Rightarrow$ Fração associada $\mathrm{a}$ compostos de alto grau de cristalinidade (fração residual).

Os extratos resultantes do fracionamento foram analisados para determinação dos teores de $\mathrm{Zn}, \mathrm{Mn}, \mathrm{Cd}$, $\mathrm{Pb}$ e $\mathrm{Cu}$ por espectrofotometria de absorção atômica com chama de ar-acetileno em aparelho VARIAN-AA600 com limites de detecção $\left(\mathrm{mg} \mathrm{kg}^{-1}\right)$ de 0,20 para $\mathrm{Zn}$; 0,2 para Mn; 0,25 para Cd; 0,10 para $\mathrm{Pb}$; 0,2 para $\mathrm{Cu}$ e limites de quantificação $\left(\mathrm{mg} \mathrm{kg}^{-1}\right)$ de 0,7 para $\mathrm{Zn} ; 0,7$ para $\mathrm{Mn} ; 0,5$ para $\mathrm{Cd} ; 0,5$ para $\mathrm{Pb} ; 0,8$ para $\mathrm{Cu}$.

De cada exemplar arbóreo, foram coletados dados referentes à profundidade das raízes, DAP (diâmetro a altura do peito), diâmetro de copa e altura das plantas. Além disso, foram coletadas amostras de raiz, caule, casca e folha para análise da concentração de $\mathrm{Zn}, \mathrm{Mn}, \mathrm{Cd}, \mathrm{Pb}$ e $\mathrm{Cu}$.

As amostras de folha, caule e casca foram coletadas com o auxílio de um facão, lavadas em água deionizada, secas em estufa com circulação de ar forçada à $65^{\circ} \mathrm{C}$ até peso constante, moídas e acondicionadas em sacos de papel. Essas amostras foram digeridas em mistura nitro-perclórica 6:1, segundo Tedesco et al. (1995) e, posteriormente, foram quantificados os teores de $\mathrm{Zn}, \mathrm{Mn}$, $\mathrm{Cd}, \mathrm{Pb}$ e $\mathrm{Cu}$ por espectrofotometria de absorção atômica.

Para a coleta de raízes foram abertas trincheiras de 1 x 1 x $1 \mathrm{~m}$, distantes aproximadamente $30 \mathrm{~cm}$ do tronco de cada árvore. Após a abertura, procedeu-se o nivelamento das paredes do perfil com auxílio de pá reta, de forma a deixá-las lisas e verticais.

Na parede da trincheira voltada ao sistema radicular de cada árvore em estudo, foi realizada a escarificação do substrato com uma faca pedológica para separar as raízes e facilitar sua coleta. As raízes foram coletadas nas camadas de $0-10 ; 10-30$ e $30-50 \mathrm{~cm}$, representando a profundidade onde foi observada maior quantidade de raízes.

As raízes foram lavadas em água corrente e colocadas em solução de $\mathrm{CaCl}_{2} 10 \mathrm{mM}$ por 10 minutos. Posteriormente, estas raízes passaram pelos mesmos procedimentos de tratamento, extração e quantificação que foram descritos no parágrafo referente à determinação dos metais pesados em tecido vegetal.

\section{RESULTADOS E DISCUSSÃO}

Os valores referentes a granulometria e aos atributos químicos do substrato, até a profundidade de $20 \mathrm{~cm}$, são apresentados na Tabela 1. Foram observadas elevadas concentrações de nutrientes, principalmente Ca e Mg, além de $\mathrm{pH}$ elevado. Provavelmente esse caráter alcalino do substrato está relacionado à sua origem marinha, visto que durante a coleta foram observados fragmentos de conchas e não houve adição de calcário na etapa inicial de revegetação.

Cada valor corresponde a média de 4 repetições \pm erro padrão

A presença de carbono orgânico se deveu à vegetação presente na área onde foram coletadas as amostras. Esta área foi revegetada em 1999 e, por ocasião da amostragem, apresentava plantas herbáceas, arbustos e árvores que adicionaram ao solo material orgânico por meio da queda de folhas, caules, frutos, além da contribuição proveniente das raízes.

Analisando a granulometria do substrato (Tabela 1), todos os pontos de coleta apresentaram textura média, de acordo com o diagrama triangular simplificado utilizado pela Empresa Brasileira de Pesquisa Agropecuária para a classificação textural do solo.

De maneira geral, as concentrações mais elevadas de metais pesados foram observadas nas maiores profundidades $(10-50 \mathrm{~cm})$, com exceção do $\mathrm{Pb}$ e $\mathrm{Cu}$

Tabela 1 - Granulometria (areia, silte e argila) e atributos químicos do substrato da área de estudo

\begin{tabular}{lc}
\hline Granulometria (areia; silte; argila) $(\%)$ & $67 ; 10 ; 23$ \\
\hline $\mathrm{pH} \mathrm{H}_{2} \mathrm{O}$ & $7,78 \pm 0,07$ \\
$\mathrm{CE}^{1}\left(\mathrm{mS} \mathrm{cm}^{-1}\right)$ & $1,90 \pm 0,18$ \\
$\mathrm{Ca}\left(\mathrm{mmol}_{\mathrm{c}} \mathrm{dm}^{-3}\right)$ & $81,8 \pm 0,59$ \\
$\mathrm{Mg}\left(\mathrm{mmol}_{\mathrm{c}} \mathrm{dm}^{-3}\right)$ & $24,5 \pm 0,16$ \\
$\mathrm{~K}\left(\mathrm{mmol}_{\mathrm{c}} \mathrm{dm}^{-3}\right)$ & $4,3 \pm 0,04$ \\
$\mathrm{Na}\left(\mathrm{mmol}_{\mathrm{c}} \mathrm{dm}^{-3}\right)$ & $6,2 \pm 0,08$ \\
$\mathrm{H}+\mathrm{Al}\left(\mathrm{mmol}_{\mathrm{c}} \mathrm{dm}^{-3}\right)$ & $1,1 \pm 0,05$ \\
$\mathrm{Al}\left(\mathrm{mmol}_{\mathrm{c}} \mathrm{dm}^{-3}\right)$ & $0,0 \pm 0,00$ \\
$\mathrm{~S}^{2}\left(\mathrm{mmol}_{\mathrm{c}} \mathrm{dm}^{-3}\right)$ & $116,8 \pm 0,73$ \\
$\mathrm{~T}^{3}\left(\mathrm{mmol}^{\mathrm{c}} \mathrm{dm}^{-3}\right)$ & $117,9 \pm 0,73$ \\
$\mathrm{~V}^{4}(\%)$ & $99 \pm 0,44$ \\
$\mathrm{n}^{5}(\%)$ & $5,2 \pm 0,44$ \\
$\mathrm{CO}^{6}\left(\mathrm{~g} \mathrm{~kg}^{-1}\right)$ & $0,94 \pm 0,07$ \\
$\mathrm{P}\left(\mathrm{mg} \mathrm{dm}^{-3}\right)$ & $18,13 \pm 2,13$ \\
\hline
\end{tabular}

${ }^{1} \mathrm{CE}$, condutividade elétrica; ${ }^{2} \mathrm{~S}$, soma de bases trocáveis; ${ }^{3} \mathrm{~T}$, capacidade de troca de cátions (CTC); ${ }^{4} \mathrm{~V}$, índice de saturação por base; ${ }^{5} \mathrm{n}$, porcentagem de saturação por sódio na $\mathrm{CTC} ;{ }^{6} \mathrm{CO}$, carbono orgânico 
(Tabela 2). Os elementos $\mathrm{Zn}, \mathrm{Mn}$ e Cd apresentam elevada mobilidade no solo (CHAVES; SOUZA; TITO., 2008; COSTA et al., 2007), o que pode ter contribuído com o acúmulo desses elementos nas maiores profundidades.

Com exceção do $\mathrm{Cd}$, as concentrações totais dos demais elementos estiveram abaixo do valor de referência de qualidade, segundo Conselho Nacional do Meio-Ambiente (2009). O cádmio apresentou, na profundidade de $10-30 \mathrm{~cm}$, concentração acima do valor de investigação, segundo Conselho Nacional do MeioAmbiente (2009), para solos agrícolas (3,0 mg kg-1).
Isso evidencia a contaminação do substrato com cádmio que, apesar de ser um elemento com elevada mobilidade no solo, teve as maiores concentrações na profundidade intermediária. Provavelmente isso aconteceu devido aos elevados valores de $\mathrm{pH}$ do solo (Tabela 2), que tende a reduzir a solubilidade de $\mathrm{Cd}$ e evitar a lixiviação.

De acordo com os resultados do fracionamento químico de metais pesados (Figura 1), maiores concentrações desses elementos foram encontradas na fração F5, que corresponde à fração química mais estável em termos de disponibilidade de metais pesados. Isso

Tabela 2 - Concentrações pseudototais de metais pesados e $\mathrm{pH}$ do substrato em diferentes profundidades

\begin{tabular}{lcccccc}
\hline Profundidade & $\mathrm{Zn}$ & $\mathrm{Mn}$ & $\mathrm{Cd}$ & $\mathrm{Pb}$ & $\mathrm{Cu}$ & $\mathrm{pH}^{(1)}$ \\
\hline $\mathrm{cm}$ & \multicolumn{5}{c}{$\mathrm{mg} \mathrm{kg}^{-1}$} \\
\hline $0-10$ & $108,475 \pm 3,29$ & $179,394 \pm 5,76$ & $2,369 \pm 0,11$ & $27,763 \pm 0,27$ & $7,778 \pm 0,37$ & $7,6 \pm 0,1$ \\
$10-30$ & $162,513 \pm 20,10$ & $234,747 \pm 13,60$ & $3,180 \pm 0,30$ & $29,697 \pm 0,78$ & $7,359 \pm 0,69$ & $7,7 \pm 0,0$ \\
$30-50$ & $169,909 \pm 11,42$ & $216,784 \pm 13,67$ & $2,500 \pm 0,16$ & $24,741 \pm 0,66$ & $5,325 \pm 0,32$ & $7,5 \pm 0,1$ \\
\hline
\end{tabular}

(1)relação solo:água 1:2,5. Cada valor corresponde a média de 4 repetições \pm erro padrão

Figura 1- Fracionamento químico de metais pesados no substrato
$\mathbf{v} 1$
口 F3
口 F4 口 F5

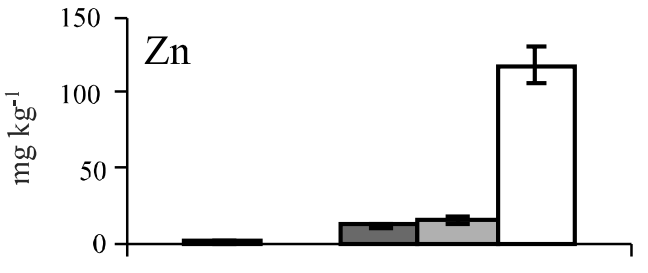

Frações

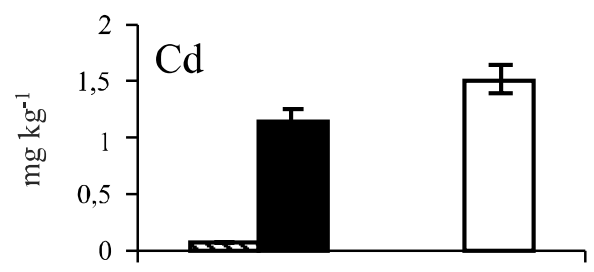

Frações

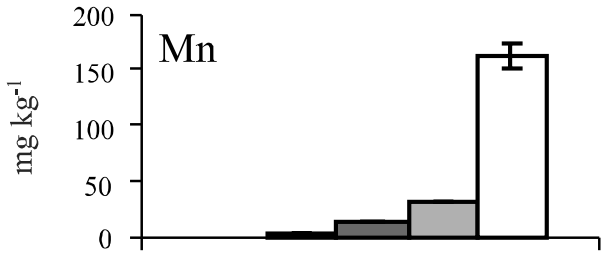

Frações

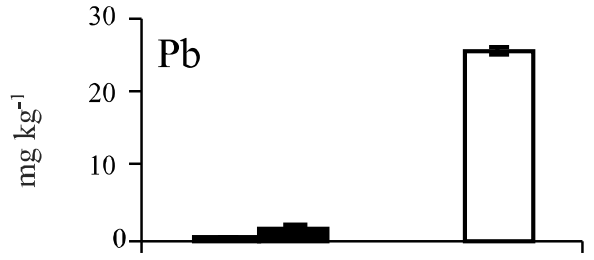

Frações

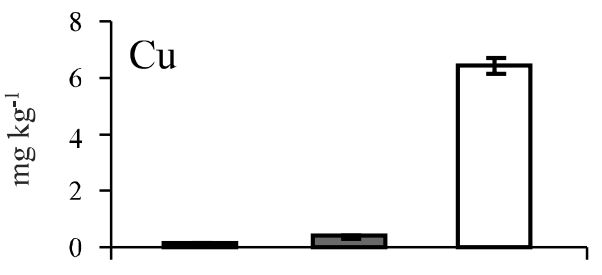

Frações 
ocorreu provavelmente, devido ao elevado $\mathrm{pH}$ do substrato (Tabela 2) que, de acordo com Trevizam et al. (2010) e Chaves, Souza e Tito (2008), é um importante parâmetro que influencia a partição do metal entre o solo e a solução solo, ou seja, na adsorção e dessorção dos metais.

F1: extração com água; $\mathrm{F} 2$ : extraído com $\mathrm{MgCl}_{2}-$ extraído com água; F3: extraído com DTPA - extraído com $\mathrm{MgCl}_{2} ; \mathrm{F} 4$ : extraído com ácido acético-extraído com $\mathrm{MgCl}_{2}$; F5: total- $\sum$ das frações (F1, F2, F3 e F4). Cada valor corresponde a média de 4 repetições \pm erro padrão.

De acordo com Agourakis et al. (2006), o pH neutro e ligeiramente alcalino favorece a precipitação e adsorção de metal no solo, diminuindo sua disponibilidade para as plantas e a lixiviação para as águas subterrâneas.

Porém, é importante ressaltar que elevadas concentrações de $\mathrm{Cd}$ foram encontradas na fração trocável F2. A fração trocável, presente na maioria dos procedimentos de extração, é considerada uma fração em que os contaminantes se apresentam com certa mobilidade e biodisponibilidade (WANG; ZHANG; JIN, 2009). Appel et al. (2008) estudando o comportamento do $\mathrm{Pb}$ e $\mathrm{Cd}$ em três solos tropicais, determinaram que o $\mathrm{Cd}$ apresentou menor afinidade com os sítios ligantes no solo, tendendo a se acumular em frações de maior biodisponibilidade.

$\mathrm{O} \mathrm{Pb}$ e o $\mathrm{Cu}$, também com elevadas concentrações na fração F5, não apresentaram comportamento semelhante ao $\mathrm{Zn}$ e Mn em relação às outras frações químicas. As concentrações de $\mathrm{Pb}$ e $\mathrm{Cu}$ nas frações $\mathrm{F} 3$ e F4 foram quase nulas, confirmando resultados de Costa e Moreira (2007) que observaram também que a mobilidade do $\mathrm{Pb}$ foi muito baixa nos solos estudados, indicando que este metal pode apresentar menor risco de contaminação do ambiente.

As espécies Córdia - africana e Acácia apresentaram maior profundidade de raízes finas e DAP em relação às demais (Tabela 3), indicando que estas espécies podem apresentar maior potencial para serem utilizadas em estratégias de fitoestabilização. Segundo
Nevel et al. (2007), a profundidade do sistema radicular e o desenvolvimento do caule, são importantes características que devem apresentar espécies a serem utilizadas em estratégias de fitoestabilização.

De acordo com Lorenzi et al. (2002), todas as espécies estudadas estão dentro dos padrões normais de desenvolvimento, com relação à altura e diâmetro de copa. Isso mostra a tolerância das espécies vegetais a áreas contaminadas com $\mathrm{Cd}$.

As concentrações de $\mathrm{Zn}, \mathrm{Pb}, \mathrm{Cu}$ e $\mathrm{Mn}$ (Tabela 4) nas espécies ficaram abaixo dos limites de concentração normal em plantas, segundo Ross (1994), para todas as partes das plantas estudadas. Com relação ao $\mathrm{Cd}$, as concentrações foram acima dos limites normais $\left(0,8 \mathrm{mg} \mathrm{kg}^{-1}\right)$, com exceção do sistema radicular da Córdia-africana, da folha da Acácia e do caule do Sabiá. No entanto, os níveis críticos de toxidade de $\mathrm{Cd}\left(5 \mathrm{mg} \mathrm{kg}^{-1}\right)$ não foram atingidos. Essa elevada concentração de $\mathrm{Cd}$ ocorreu provavelmente devido às elevadas concentrações desse elemento na fração F2 (Figura 1). De acordo com Nevel et al. (2007), o teor e o acúmulo do elemento nos tecidos das plantas ocorrem em função da sua disponibilidade na solução do solo e os teores nas raízes e na parte aérea aumentam com a elevação da concentração de elementos na solução do solo.

Em relação às raízes, maiores teores de metais foram encontrados, em geral, na profundidade de $0-10 \mathrm{~cm}$, embora não tenha ocorrido diferença significativa para a maior parte dos metais pesados e plantas estudadas. Isso ocorreu provavelmente, por ser a região com maiores quantidades de raízes finas (Tabela 3), consideradas como mais eficientes em relação à absorção (BRUNNER et al., 2008).

As espécies arbóreas estudadas apresentaram respostas distintas em relação aos teores de metais pesados em suas diferentes partes (Tabela 4). Na espécie Córdia-africana as maiores concentrações de $\mathrm{Zn}$ e $\mathrm{Pb}$ foram encontradas nas folhas. Além disso, $\mathrm{Cd}$ e $\mathrm{Cu}$ tenderam a se acumular mais na parte aérea da planta

Tabela 3 - Características morfológicas das plantas estudadas

\begin{tabular}{lccccc}
\hline \multirow{2}{*}{ Espécie } & DAP* & Altura & Diâmetro de copa & Prof. raízes finas & Prof. raízes grossas \\
\cline { 2 - 6 } & $(\mathrm{cm})$ & $(\mathrm{m})$ & $\left(\mathrm{m}^{2}\right)$ & & $(\mathrm{cm})$ \\
\hline Córdia africana & $21,8 \pm 0,5$ & $5,1 \pm 0,1$ & $9,0 \pm 0,0$ & $28 \pm 0,1$ & $49 \pm 0,1$ \\
Sabiá & $18,0 \pm 0,7$ & $4,1 \pm 0,1$ & $6,5 \pm 0,5$ & $23 \pm 0,2$ & $49 \pm 0,0$ \\
Acácia & $21,5 \pm 1,3$ & $4,9 \pm 0,1$ & $5,8 \pm 0,3$ & $26 \pm 0,1$ & $50 \pm 0,1$ \\
Angico branco & $17,0 \pm 0,7$ & $4,6 \pm 0,1$ & $8,5 \pm 0,3$ & $25 \pm 0,0$ & $49 \pm 0,1$ \\
\hline
\end{tabular}

*DAP: Diâmetro a altura do peito. Cada valor corresponde a média de 4 repetições \pm erro padrão 
Tabela 4 - Teores de metais pesados $\left(\mathrm{mg} \mathrm{kg}^{-1}\right)$ nas diferentes partes das plantas desenvolvidas em substrato contaminado

\begin{tabular}{|c|c|c|c|c|c|}
\hline Partes da planta & $\mathrm{Zn}$ & $\mathrm{Mn}$ & $\mathrm{Cd}$ & $\mathrm{Pb}$ & $\mathrm{Cu}$ \\
\hline \multicolumn{6}{|c|}{ Córdia - africana } \\
\hline Caule & $21,312 \pm 2,37 \mathrm{cB}$ & $11,175 \pm 1,43 \mathrm{aA}$ & $1,200 \pm 0,16 a A$ & $2,930 \pm 0,94 \mathrm{aB}$ & $7,580 \pm 1,52 \mathrm{aA}$ \\
\hline Casca & $19,010 \pm 2,89 \mathrm{cB}$ & $15,425 \pm 1,10 \mathrm{aA}$ & $1,085 \pm 0,23 \mathrm{aA}$ & $4,502 \pm 1,99 \mathrm{aB}$ & $11,090 \pm 0,29 \mathrm{aA}$ \\
\hline Folha & $48,370 \pm 4,15 \mathrm{aA}$ & $29,787 \pm 2,72 b A$ & $1,400 \pm 0,05 \mathrm{aA}$ & $10,592 \pm 3,31 \mathrm{aA}$ & $7,717 \pm 1,32 \mathrm{aA}$ \\
\hline Raiz0-10 cm & $17,007 \pm 4,00 \mathrm{aB}$ & $48,930 \pm 11,54 \mathrm{aA}$ & $0,487 \pm 0,42 \mathrm{bB}$ & $1,585 \pm 1,37 \mathrm{aB}$ & $3,307 \pm 1,01 \mathrm{aB}$ \\
\hline Raiz10-30 cm & $3,160 \pm 1,83 \mathrm{aC}$ & $38,245 \pm 18,54 \mathrm{aA}$ & $0,000 \pm 0,00 \mathrm{bB}$ & $0,415 \pm 0,21 \mathrm{aB}$ & $1,050 \pm 0,70 \mathrm{aB}$ \\
\hline Raiz30-50 cm & $12,840 \pm 7,43 \mathrm{bB}$ & $27,775 \pm 16,07 \mathrm{aA}$ & $0,200 \pm 0,17 \mathrm{bB}$ & $1,332 \pm 0,60 \mathrm{aB}$ & $2,727 \pm 2,05 \mathrm{aB}$ \\
\hline \multicolumn{6}{|c|}{ Sabiá } \\
\hline Caule & $8,445 \pm 1,20 \mathrm{~dB}$ & $12,475 \pm 1,92 \mathrm{aB}$ & $0,705 \pm 0,17 \mathrm{aB}$ & $1,527 \pm 0,63 \mathrm{aA}$ & $2,275 \pm 0,34 \mathrm{aA}$ \\
\hline Casca & $12,212 \pm 1,49 \mathrm{cB}$ & $18,085 \pm 1,92 \mathrm{aB}$ & $0,955 \pm 0,17 \mathrm{aB}$ & $1,007 \pm 0,55 \mathrm{aA}$ & $2,577 \pm 0,36 \mathrm{aA}$ \\
\hline Folha & $23,262 \pm 1,68 \mathrm{bA}$ & $26,397 \pm 2,48 b B$ & $0,887 \pm 0,11 \mathrm{bB}$ & $2,592 \pm 0,41 \mathrm{bA}$ & $1,590 \pm 0,14 \mathrm{aB}$ \\
\hline Raiz0-10 cm & $12,697 \pm 2,58 \mathrm{aB}$ & $53,430 \pm 14,28 \mathrm{aA}$ & $4,170 \pm 0,18 \mathrm{aA}$ & $0,582 \pm 0,37 \mathrm{aA}$ & $2,972 \pm 0,30 \mathrm{aA}$ \\
\hline Raiz10-30 cm & $4,820 \pm 0,34 \mathrm{aB}$ & $23,962 \pm 5,24 \mathrm{aB}$ & $3,085 \pm 0,58 \mathrm{aA}$ & $0,075 \pm 0,06 \mathrm{aA}$ & $1,417 \pm 0,30 \mathrm{aB}$ \\
\hline Raiz30-50 cm & $11,100 \pm 1,95 b B$ & $32,590 \pm 5,06 \mathrm{aB}$ & $4,667 \pm 1,08 \mathrm{aA}$ & $1,542 \pm 0,84 \mathrm{aA}$ & $1,890 \pm 0,30 \mathrm{aB}$ \\
\hline \multicolumn{6}{|c|}{ Acácia } \\
\hline Caule & $33,855 \pm 2,23 \mathrm{bA}$ & $6,277 \pm 1,45 \mathrm{aC}$ & $1,032 \pm 0,18 \mathrm{aB}$ & $0,902 \pm 0,39 \mathrm{aA}$ & $3,620 \pm 0,36 \mathrm{aA}$ \\
\hline Casca & $26,975 \pm 1,65 \mathrm{bA}$ & $11,020 \pm 3,19 \mathrm{aC}$ & $0,872 \pm 0,15 \mathrm{aB}$ & $0,120 \pm 0,10 \mathrm{aA}$ & $1,857 \pm 0,26 \mathrm{aA}$ \\
\hline Folha & $41,075 \pm 1,82 \mathrm{aA}$ & $44,170 \pm 2,15 \mathrm{aA}$ & $0,750 \pm 0,07 \mathrm{bB}$ & $0,202 \pm 0,11 \mathrm{bA}$ & $3,520 \pm 0,45 \mathrm{aA}$ \\
\hline Raiz0-10 cm & $14,377 \pm 1,95 \mathrm{aB}$ & $26,465 \pm 4,80 \mathrm{aB}$ & $4,495 \pm 0,40 \mathrm{aA}$ & $1,625 \pm 0,81 \mathrm{aA}$ & $4,820 \pm 2,14 \mathrm{aA}$ \\
\hline Raiz10-30 cm & $6,535 \pm 1,55 \mathrm{aB}$ & $12,895 \pm 3,60 \mathrm{aC}$ & $2,417 \pm 0,33 \mathrm{aB}$ & $0,050 \pm 0,04 \mathrm{aA}$ & $1,400 \pm 0,39 \mathrm{aA}$ \\
\hline Raiz30-50 cm & $32,037 \pm 6,14 \mathrm{aA}$ & $18,317 \pm 9,66 \mathrm{aC}$ & $5,675 \pm 1,25 \mathrm{aA}$ & $0,075 \pm 0,06 \mathrm{aA}$ & $5,702 \pm 2,39 \mathrm{aA}$ \\
\hline \multicolumn{6}{|c|}{ Angico branco } \\
\hline Caule & $45,492 \pm 2,80 \mathrm{aA}$ & $12,097 \pm 3,60 \mathrm{aB}$ & $0,877 \pm 0,12 \mathrm{aA}$ & $0,517 \pm 0,37 \mathrm{aA}$ & $5,380 \pm 0,70 \mathrm{aA}$ \\
\hline Casca & $40,560 \pm 4,48 \mathrm{aA}$ & $20,710 \pm 4,83 \mathrm{aB}$ & $0,967 \pm 0,08 \mathrm{aA}$ & $1,187 \pm 0,27 \mathrm{aA}$ & $4,622 \pm 1,47 \mathrm{aA}$ \\
\hline Folha & $39,795 \pm 2,29 \mathrm{aA}$ & $24,062 \pm 1,42 \mathrm{bB}$ & $0,960 \pm 0,11 \mathrm{bA}$ & $1,162 \pm 0,65 \mathrm{bA}$ & $7,445 \pm 0,17 \mathrm{aA}$ \\
\hline Raiz0-10 cm & $26,050 \pm 6,72 \mathrm{aB}$ & $31,117 \pm 7,04 \mathrm{aA}$ & $1,382 \pm 0,64 \mathrm{bA}$ & $0,850 \pm 0,04 \mathrm{aA}$ & $4,467 \pm 0,65 \mathrm{aA}$ \\
\hline Raiz10-30 cm & $9,180 \pm 1,33 \mathrm{aC}$ & $13,067 \pm 3,42 \mathrm{aB}$ & $0,515 \pm 0,35 \mathrm{bA}$ & $0,132 \pm 0,07 \mathrm{aA}$ & $2,515 \pm 0,41 \mathrm{aA}$ \\
\hline Raiz30-50 cm & $8,670 \pm 2,21 b C$ & $13,082 \pm 2,21 \mathrm{aB}$ & $0,717 \pm 0,57 \mathrm{bA}$ & $0,050 \pm 0,04 \mathrm{aA}$ & $3,150 \pm 0,88 \mathrm{aA}$ \\
\hline
\end{tabular}

Cada valor corresponde a média de 4 repetições \pm erro padrão. Valores seguidos da mesma letra minúscula para plantas dentro de cada parte da planta e metal; e maiúsculas para partes da planta dentro de uma mesma planta e metal, não diferem entre si ao nível de 5\% pelo teste de Scott-Knott

em comparação com a raiz, mostrando que essa espécie apresenta alta capacidade de translocação dos metais absorvidos. Espécies com essa característica podem facilitar o processo de distribuição de metais pesados nos componentes do ecossistema, tendo em vista que as folhas reciclam mais rapidamente que as demais partes da planta (MERTENS et al., 2007). Esses resultados foram semelhantes ao observado por Mertens et al. (2004) que, ao estudarem cinco espécies arbóreas desenvolvidas em sedimento contaminado com metais pesados, encontraram elevadas concentrações de Cd e Zn nas folhas de Populus alba L., indicando risco de utilização dessa espécie em programas de fitoestabilização.

Com exceção da Córdia - africana, o Cd acumulouse nas raízes das plantas. Segundo Pulford e Watson (2003), a regulação da absorção de metais pesados na rizosfera, o acúmulo desses nas raízes (desde que preservada sua integridade e funções primárias) e a baixa translocação para a parte aérea são considerados mecanismos pelos quais o sistema radicular pode contribuir para a tolerância de espécies a metais pesados. 


\section{CONCLUSÕES}

1. A concentração de cádmio no substrato está acima do valor de investigação segundo legislação CONAMA, evidenciando contaminação. Além disso, elevadas concentrações desse elemento foram encontradas na fração trocável F2, evidenciando uma certa mobilidade e biodisponibilidade de Cd no substrato;

2. As espécies vegetais apresentaram tolerância a áreas contaminadas com $\mathrm{Cd}$, que tendeu a se acumular nas raízes das plantas, com exceção da Córdia-africana.

\section{AGRADECIMENTOS}

À Capes e Faperj pela concessão de bolsa de estudo à primeira autora.

\section{REFERÊNCIAS}

ASSOCIAÇÃO BRASILEIRA DE NORMAS TÉCNICAS. NBR-10004: Resíduos sólidos - Classificação. Rio de Janeiro, 2004. $71 \mathrm{p}$.

AGOURAKIS, D. C. et al. Comportamento de Zn e Mn de pilhas alcalinas em uma coluna de solo. Química Nova, v. 29, n. 05 , p. 960-964, 2006.

APPEL, C. et al. Sequential sorption of lead and cadmium in three tropical soils. Environmental Pollution, v. 155, n. 01, p. 132-140, 2008.

BRUNNER, I. et al. Heavy metal accumulation and phytostabilisation potential of tree fine roots in a contaminated soil. Environmental Pollution, v. 152, n. 03, p. 559-568, 2008.

CHAVES, L. H. G.; SOUZA, R. S. de; TITO, G. A. Adsorção de zinco em Argissolos do Estado da Paraíba: efeito do pH. Revista Ciência Agronômica, v. 39, n. 04, p. 511-516, 2008.

CONSELHO NACIONAL DO MEIO AMBIENTE. Resolução $n^{0}$ 420, de 28 de dezembro de 2009. Disponível em: http:// www.fundagres.org.br/biossolido/images/downloads/resolucao_ 420_2009.pdf>. Acesso em: 9 set. 2011.

COSTA, C. N. et al. Fracionamento sequencial de cádmio e chumbo em solos. Ciência Rural, v. 37, n. 05, p. 1323-1328, 2007.

COSTA, D. G.; MOREIRA, A. Dinâmica da matéria orgânica na recuperação de clareiras da floresta amazônica. Pesquisa Agropecuária Brasileira, v. 39, n. 10, p. 1013-1019, 2007.

EMPRESA BRASILEIRA DE PESQUISA AGROPECUÁRIA Manual de métodos de análise de solo. 2. ed. Rio de Janeiro: EMBRAPA, 1997. 212 p.
KAVAMURA, V. N.; ESPOSITO, E. Biotechnological strategies applied to the decontamination of soils polluted with heavy metals. Biotechnology Advances, v. 28, n. 01, p. 61-69, 2010.

LORENZI, H. Árvores Brasileiras. Nova Odessa: Plantarum, 2002. $351 \mathrm{p}$.

MERTENS, J. et al. Tree species effect on the redistribution of soil metals. Environmental Pollution, v. 149, n. 02, p. 173-181, 2007.

MERTENS, J. et al. Metal uptake by young trees from dredged brackish sediment: limitations and possibilities for phytoextraction and phytostabilisation. Science of the Total Environment, v. 326, n. 01, p. 209-215, 2004.

NEVEL, L. V. et al. Phytoextraction of metals from soils: How far from practice? Environmental Pollution, v. 150, n. 01, p. 34-40, 2007.

PAQUIN, D. G.; CAMPBELL, S.; LI, Q. X. Phytoremediation in subtropical Hawaii a review of over 100 plant species. Remediation Journal, v. 14, n. 02, p. 127-139, 2004.

PILON-SMITS, E. Phytoremediation. Annual Review of Plant Biology, v. 56, n. 01, p. 15-39, 2005.

PULFORD, I. D.; WATSON, C. Phytoremediation of heavy metal-contaminated land by trees a review. Environment International, v. 29, n. 04, p. 529-540, 2003.

ROSS, S. M. Toxic metals in soil-plant systems. New York: John Willey \& Sons, 1994. 496 p.

SANTOS, F. S. et al. Chemical amendment and phytostabilization of an industrial residue contaminated with $\mathrm{Zn}$ and $\mathrm{Cd}$. Scientia Agricola, v. 64, n. 05, p. 506-512, 2007.

SCOTT, K. Cause and control of losses of chromium during nitric-perchloric acid oxidation of aquatic sediments. Analyst, v. 103 , p. $754-758,1978$.

TEDESCO, M. J. et al. Análise de solo, plantas e outros materiais. 2. ed. Porto Alegre: Departamento de Solos/UFRGS, 1995. 174 p. (Boletim técnico n ${ }^{\circ}$ 5).

TREVIZAM, A. R. et al. Aplicação de fósforo para imobilização química do cádmio em solo contaminado. Química Nova, v. 33, n. 06, p. 1235-1241, 2010.

WANG, J.; ZHANG, C. B.; JIN, Z. X. The distribution and phytoavailability of heavy metal fractions in rhizosphere soils of Paulowniufortunei (seem) Hems near a Pb/Zn smelter in Guangdong, PR China. Geoderma, v. 148, n. 03, p. 299-306, 2009.

XIAO, X. et al. Potential of Pteris vittata L. for phytoremediation of sites co-contaminated with cadmium and arsenic: the tolerance and accumulation. Journal of Environmental Sciences, v. 20, n. 01, p. 62-67, 2008. 